\title{
Pucallpa - Portrait einer Pionierstadt in der peruanischẹn Selva (Ostperu)
}

\section{Naturraum und Besiedlung}

Weit ausladende, braune Schleifen durch den immergrünen Teppich ziehend, fließt der Rio Ucayali träge dahin. In den vielen Hunderten und Tausenden von Jahren, in denen er schon über viele Hunderte von Kilometern $(1900 \mathrm{~km})$ von Süden gegen Norden dem Amazonas zuströmt, hat er tiefe und markante Spuren seines Waltens in dieser Landschaft hinterlassen. In seinem Mittellauf beträgt das Gefälle nur $5 \mathrm{~cm}$ auf einen Kilometer. Hier nimmt sich der Ucayali, gespiesen von zahlreichen Zuflüssen aus den Anden, genügend Zeit, sein mitgeführtes Material abzulagern. Schon oft hat er sich dadurch sein eigenes Bett verbaut und sich ein neues schaffen müssen. Daher finden sich auf einer Breite von mehreren Kilometern die Spuren seines Wirkens: Mäander, kilometerlange Lagunen (Alt- oder Totwässer), Sümpfe, Tümpel, ausgetrocknete Flußschlingen, Sandbänke, Auenwälder; der Boden ist durch das geprägt, was der Ucayali abgelagert hat: vor allem Kiesel, Lehm, Sand.

Solche braunen, mäandrierenden Flüsse und Seenund Sumpflandschaften sind typisch für die Mittelläufe der zahllosen Flüsse des Amazonastieflandes. Der Begriff «Tiefland» ist eigentlich irreführend, ist dieses Gebiet doch vielmehr durch das Wasser als durch das Festland geprägt, obwohl man vom Flugzeug aus den Eindruck eines zusammenhängenden grünen Teppichs erhält. Das Vorherrschen des Wassers ist besonders während der Regenzeit (Oktober bis Mai) augenfällig, wenn der Wasserspiegel um 10 bis $20 \mathrm{~m}$ über den Wasserstand der Trockenzeit (Juni bis September) ansteigt und dadurch große Gebiete überschwemmt werden und unter Wasser stehen. Durch diese Überschwemmungen bilden sich zahllose kleine Verbindungskanäle, die es den Einwohnern ermöglichen, etwa mit dem Kanu weite Flußschlingen abzukürzen, um damit große Distanzen zwischen den Dörfern und den Marktzentren erheblich zu verringern. In einer solchen Landschaft und bei feucht-heißem Klima siedeln also die Bewohner des Mittleren Rio Ucayali. Da sie (vor allem Indios und Mestizen) in ihrer Lebensweise aufs Wasser ausgerichtet sind (Fischen, Verkehr), benutzen sie häufig Flußterrassen für die Anlage von Siedlungen. In der Trockenzeit, wenn der Wasserspiegel um 10 bis 20 m niedriger ist und die Flüsse ihre Sandbänke freigeben, errichten sie sogar temporäre Siedlungen unmittelbar am Was- ser. Dauersiedlungen liegen im Amazonastiefland jedoch auf jenen Flußterrassen, die auch in der Regenzeit nicht überflutet werden können.

Eine solche Landschaft, wie wir sie am Mittellauf des Ucayali antreffen, ist in der Regel eher siedlungsfeindlich: Es werden nicht nur große Gebiete periodisch überschwemmt oder sind dauernd versumpft (Brutund Heimstätte von Myriaden von Moskitos), es ändern nicht nur viele Flüsse immer wieder ihren Lauf, sondern die Siedlungen und die dazu nötige Infrastruktur (Straßen, Eisenbahnen) sind buchstäblich auf «Sand» gebaut.

\section{Pucallpa - die aus Staub Geborene}

Pucallpa, die Pionierstadt, liegt in einer solchen Landschaft. Ihr Name kommt aus dem Quechua und bedeutet «rotes Ufer» oder «rote Klippe». $\mathrm{Zu}$ unserem Stichwort «Pucallpa» assoziierte unser Taxichauffeur in Cuzco im Andenhochland zuallererst «mucho polvo» («sehr viel Staub»). Den Uneingeweihten mag das erstaunen, denn wer assoziiert schon beim Stichwort «Urwald» oder «grüne Hölle» gerade «Staub». Und dennoch ist der Staub, besonders während der Trockenzeit, das Charakteristikum von Pucallpa. Wird nämlich in dieser Gegend die Pflanzendecke (Auenwälder, Urwald) gerodet, tritt der sandige Untergrund hervor, der früher von den Flüssen abgelagert wurde. Da die Straßen in und um Pucallpa herum nicht ásphaltiert sind, übernimmt der Staub die Herrschaft über die Stadt. Er ist so fein, daß er, durch Wind und Straßenverkehr aufgewirbelt und in riesige Staubwolken verwandelt, durch alle Ritzen und Poren eindringt. Der Staub durchzieht die Stadt wie ein herbstlicher Morgennebel und legt jeden Tag eine neue dicke Schicht über alles, was sich in der Stadt befindet. Die Bewohner der Stadt sind deshalb froh, wenn kleinere Regengüsse diese Plage wenigstens für ein paar Stunden vergessen lassen. Nur das «Grand Hotel» kann es sich leisten, wenn es einige Tage nicht geregnet hat, das Stückchen Hauptstraße, das um das Haus herum führt, mehrmals täglich mit Wasser abzuspritzen, um gerade die allergrößten Staublawinen zurückzuhalten.

Heinz Joos-Süßtrunk, lic. phil. I, Hauptstraße 69, 4107 Ettingen. 


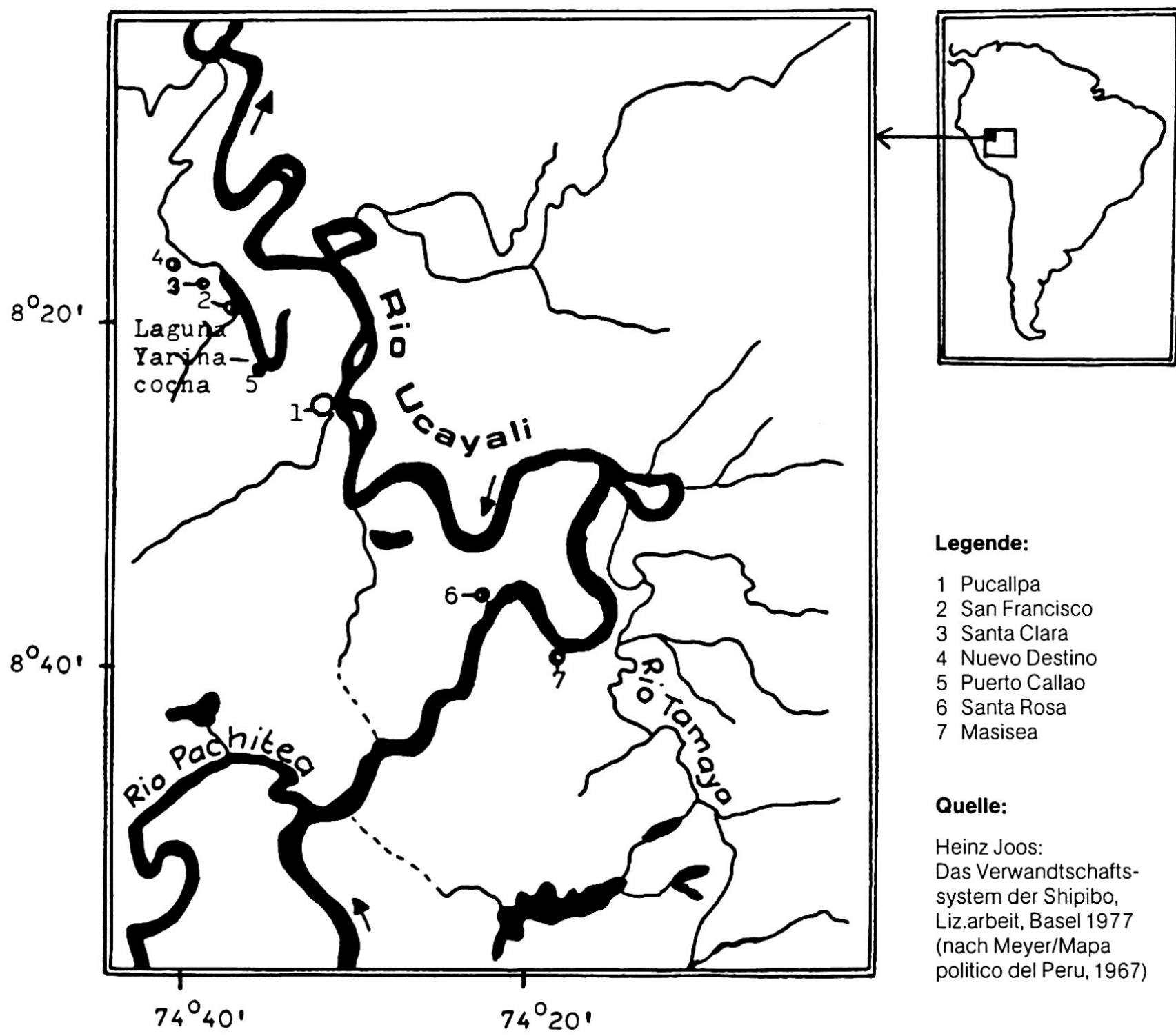

Wie sehr Regengüsse in der Trockenzeit auch willkommen sind, so sehr haben die gewitterhaften, tropischen Sturzfluten auch ihre Nachteile: Der Staub verwandelt sich in Schlamm. Und so verwandeln sich die Staubpisten Pucallpas schon nach kurzer Zeit in unpassierbaren Morast. In der Trockenzeit sind die Straßen nach zwei oder drei Tagen wieder «normal», das heißt mit einer Geschwindigkeit von $5 \mathrm{~km} / \mathrm{h}$, befahrbar, in der Regenzeit ist jedoch jeglicher Verkehr unmöglich.

\section{Pionierstadt zwischen Improvisation und Planung}

Aber nicht nur der Staub, sondern ein lebendiger, aber auch harter Pioniergeist beherrscht die Stadt. So wie die Stadt buchstäblich auf «Sand» gebaut ist, so könnte man im übertragenen Sinne sagen, die Stadt sei nicht für die Zukunft, sondern ausschließlich für die Gegenwart gebaut worden. Sie hat etwas Zufälliges, Improvisiertes, Vorläufiges, noch in Entwicklung Begriffenes, zugleich aber - all dem widersprechend - etwas sehr wohl Durchdachtes an sich: In Pucallpa leben heute nach inoffiziellen Schätzungen etwa 70000 Menschen (vor allem Mestizen, Kreolen, Indios). Bis 1967 gab es weder Kanalisationen noch Trinkwasser. Die StraBen sind bis heute nicht asphaltiert. Über diese holprigen Staubpisten, die mehr frisch gepflügten Äckern gleichen als Orten der Fortbewegung, kriechen bei $5 \mathrm{~km} / \mathrm{h}$ Personen- und Lastautos aller nur erdenklichen und vor allem unerdenklichen Marken und Jahrgänge und scheinbar "selbstgebastelte» Autobusse und "colectivos». Die Trottoirs, normalerweise Orte des Gehens, machen, wenn es sie überhaupt gibt, den gleichen desolaten Eindruck. Die Bauweise der Häuser wechselt von Bretterbuden mit Wellblechdächern zu ein- oder zweistöckigen, nur einseitig, auf der der Straße zugewandten Fassade bemalten Backsteinoder - ausnahmsweise - Betonbauten. Sanitär- und elektrische Installationen (Röhren, Leitungen) verlaufen an den Außenwänden der Häuser und es scheint, als ob sich dabei jeder Hausbesitzer nach seinen eigenen Bedürfnissen und seinem Gutdünken, 


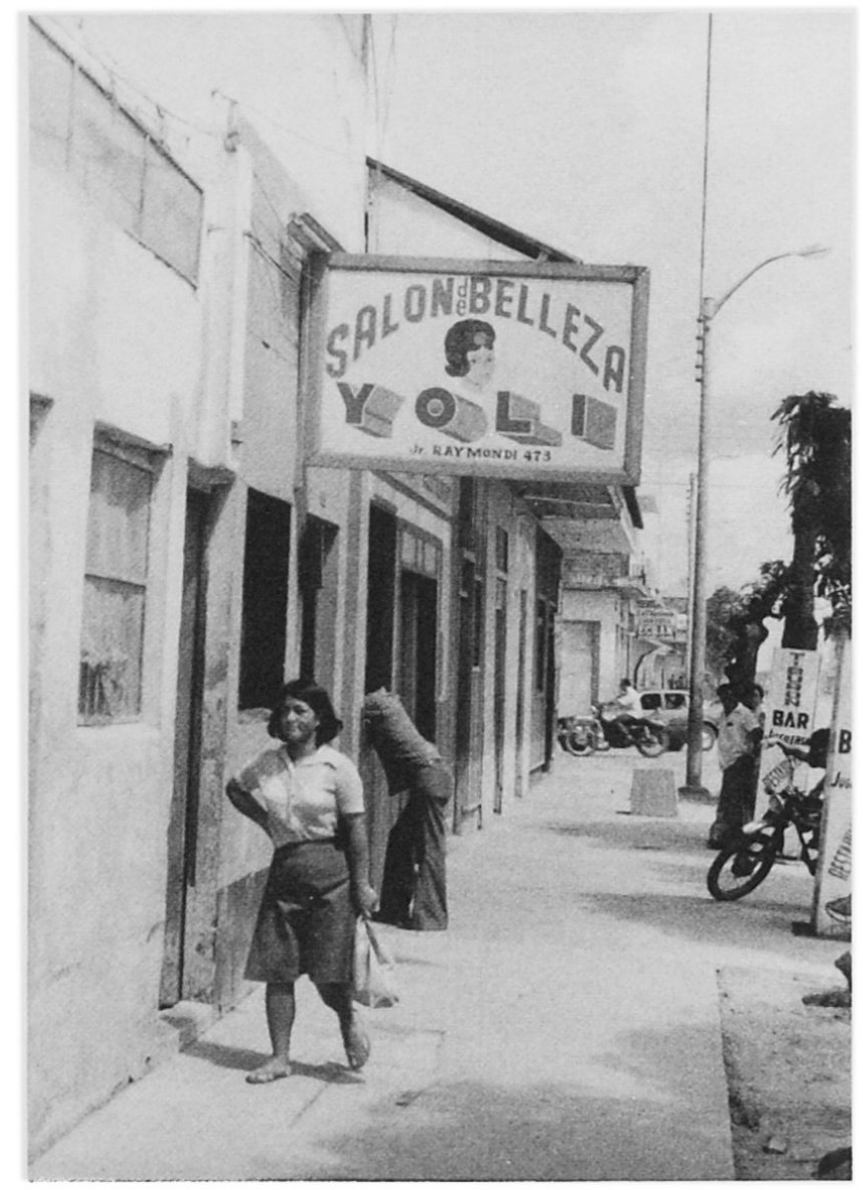

Abb. 2 Ist dieser Wunsch der Weiblichkeit, sich schön zu machen, in dieser aus Staub geborenen Stadt mitten im Amazonasdschungel, nicht erst recht legitim?

und nicht nach irgendwelchen Normen einrichtet. Wohn- und Geschäftsgebäude wechseln sich ab, dazwischen Häuser, in denen beide Funktionen untergebracht sind.

Die Geschäftsräumlichkeiten der zahlreichen kleinen Läden gleichen in ihrem mediterranen Basarstil eher einer Reihe von Garagen. Dieser Eindruck wird noch dadurch verstärkt, daß sie mehrheitlich tatsächlich nur aus einem einzigen Raum mit nur einer einzigen Öffnung, dem "Garagenton», bestehen. Man muß sich allerdings hüten, einfach von der Bauweise der Häuser auf die Qualität der angebotenen Waren und Dienstleistungen oder sogar auf den sozialen Status des Besitzers zu schließen, denn sonst kann man einige Überraschungen erleben: In Bretterbuden finden sich etwa eine "farmacia», ein "dentista», ein «salon de belleza», ein "foto estudio» usw. Man kann dabei höchstens vermuten, daß der betreffende Geschäftsinhaber noch nicht allzu lange in der Stadt ist, und daß er sich vielleicht nach einer gewissen Anfangsphase ein besseres, d. h., stabileres Haus bauen wird. Wie zufällig, improvisiert das Stadtbild Pucallpas nach einem ersten Eindruck auch scheinen mag, so Das Schachbrettschema bildet das Grundprinzip des kolonialspanischen Städtebaues. Die Idee zu diesem Grundriß fanden die spanischen Konquistadoren, wie stellt man nach genauerer Beobachtung und einem Blick auf den Stadtplan fest, daß die Anlage der Siedlung doch nicht ganz dem Zufall überlassen wurde: Das Schachbrettschema beherrscht den Grundriß: Parallele, senkrecht sich schneidende Straßenzüge; die Straßen sind breit und die Straßenflucht ist in Quadras (Blocks) aufgeteilt; ein oder mehrere Quadras sind für die Anlage von Plätzen (plazas), Pärken (parques) und Märkten (mercados) ausgespart; die Bebauung ist großzügig und flächenhaft, das heißt, man spart nicht mit Raum für die einzelnen Blocks (quadra); die einzelnen Häuser sind ein- oder zweistöckig.

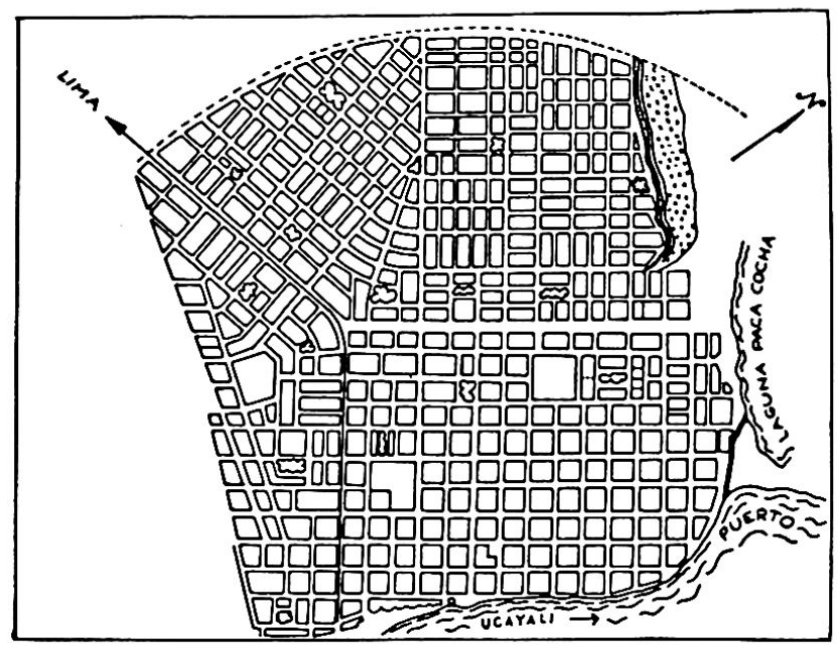

Abb. 3 «Das Schachbrettschema beherrscht den Grundriß Pucallpas: Parallele, senkrecht sich schneidende Straßenzüge; die Straßen sind breit und die Straßenflucht ist in Quadras (Blocks) aufgeteilt; die Bebauung ist großzügig und flächenhaft, das heißt man spart nicht mit Raum für die einzelnen Blocks; die einzelnen Häuser sind ein- oder zweistöckig." Dem Stadtbild Pucallpas fehlen viele der typisch kolonialspanischen, architektonischen Eigenheiten. Es fehlen überhaupt markante, visuelle Fixpunkte, an denen man sich orientieren könnte. Pucallpa ist für das Auge sehr nüchtern und monoton.

HERBERT WILHELMY1) schreibt, weder in der Neuen Welt noch in Spanien selbst, sondern sie griffen im 16. Jahrhundert, wo ja in Europa die Renaissance herrschte, auf die Schriften des römischen Stadt- und Kriegsbaumeisters VITRUVIUS POLLIO zurück, der im 1. Jahrhundert v. Chr. unter CÄSAR und AUGUSTUS wirkte. VITRUVIUS wiederum hatte in seiner Schrift «de architectura» seine eigenen Vorstellungen und jene der griechischen Architekten verarbeitet, insbesondere hatte er sich mit dem griechischen Stadtbaumeister HIPPODAMOS VON MILET beschäftigt, der im 5. Jh. v. Chr. den Schachbrettplan erfunden hat.

Für die Anlage neuer Städte in späteren Jahrhunderten hielt man in Spanisch-Amerika an diesem Grundriß fest. Und so ist es denn auch nicht verwunderlich, $\mathrm{da} ß$ man auch bei der Erschließung des tropischen Regenwaldes, die ja durchaus im Geiste der Konquista vor sich ging und geht und die Fortsetzung davon ist, bei der Anlage neuer «Kolonial»-Städte das Schach- 
brettschema verwendete und noch immer verwendet. Betrachtet man den Stadtplan von Pucallpa, so fällt auf, daß, wo es aus topographischen Gründen möglich war, ein Teil der Stadt diagonal zu den Hauptwindrichtungen angelegt ist. Ist es Zufall oder wirken hier nicht - vermittelt natürlich durch die Bauvorschriften PHILIPPS II von 1573 - die Anweisungen VITRUVIUS' nach? In dessen erstem Buch (Kap. VI, 8) heisst es nämlich: «Laßt Straßen und Gassen der Mittellinie zwischen zwei Hauptwindrichtungen folgen. Auf diese Weise werden Hausfronten und Wohnungen vor den unangenehmen Wirkungen des Windes geschützt. Denn wenn die Straßen genau in der Windrichtung verlaufen, fegen die Stürme ... mit voller Kraft durch sie hindurch. Die Hausfronten müssen daher jenen Richtungen abgewandt sein, aus denen die Winde wehen, damit diese sich an den Hausecken brechen.» (zit. n. H. WILHELMY.)

Bei der Betrachtung der Physiognomie Pucallpas fällt weiter auf, daß viele der typisch kolonialspanischen, architektonischen Eigenheiten fehlen, etwa eine das Stadtbild dominierende zentrale Plaza, die normalerweise von imposanten Kathedralen und Regierungspalästen umgeben ist. Im Weichbild der Stadt fehlen überhaupt jene markanten, visuellen Fixpunkte, die normalerweise die Aufmerksamkeit auf sich ziehen und an denen man sich orientieren kann. Pucallpa ist gekennzeichnet durch eine profane Nüchternheit und ist damit für das Auge geradezu monoton.

\section{Die Träger des Pioniergeistes}

Das Straßenbild wird vor allem von Kreolen (Nachkommen der spanischen Eroberer) und Mestizen (Mischlingen zwischen Kreolen und Indios) geprägt. Sie sind die eigentlichen Träger des Pioniergeistes. Ein Blick in die Geschäfte und Büros von Banken und Verwaltungen usw. zeigt, daß dort die Kreolen dominieren, die Mestizen führen eher einfachere, untergeordnete und damit geringer entlöhnte Arbeiten aus. Die Mestizen dominieren in der Stadt vor allem das Kleingewerbe, den Straßenhandel und die freien, offenen Märkte. Sonst stellen die Mestizen den Hauptanteil der «colonos», der Kolonisten, in Ostperu.

Die Kreolen und die Mestizen (einschließlich der Indios) orientieren sich wirtschaftlich - und natürlich auch kulturell und politisch - an unterschiedlichen Leitbildern: Die Kreolen, als Mittel- und Oberschicht des Landes, sind vorwiegend nach Lima (der Hauptstadt Perus) orientiert und deshalb bei ihrem Austausch und der Versorgung mit Gütern und Dienstleistungen von der Küste (Lima) abhängig. Die Mestizen und Indios (als Unterschicht und Parias) dagegen versorgen sich größtenteils aus der Umgebung und sind daher wirtschaftlich gegenüber dem Zentrum Lima viel unabhängiger. Diese größere Unabhängigkeit zeigt sich sehr deutlich, wenn etwa irgendeine Gewerkschaft in Lima einen Streik ausruft, dann sind in Pucallpa die Ge- schäfte und Büros der Kreolen betroffen und geschlossen. Die offenen, freien Märkte, der Kleinhandel und das Kleingewerbe der Mestizen und Indios dagegen funktionieren reibungslos weiter.

Die Mestizen orientieren sich in ihren Wünschen und Bedürfnissen zwar an der Oberschicht, doch da sie über eine schlechtere Ausbildung verfügen und sich daher beruflich weniger qualifizieren können und geringere Einkommen beziehen, sind ihren Chancen, die geweckten Bedürfnisse auf die gleiche Weise wie die Oberschicht zu befriedigen, Grenzen gesetzt. Sichtbarer Ausdruck davon sind etwa die «selbstgebastelten» und ausrangierten Lastwagen und "colectivos». (Ein "colectivo" ist eine Art Taxi, da aber gleichzeitig mehrere Personen inklusive deren Hausrat mitfahren, sind die Tarife billiger als jene der Taxis.)

Die Indios (vor allem die Shipibo und Conibo) treten in der Stadt weniger in Erscheinung - auch wirtschaftlich. Es sind vor allem die Frauen, die mit ihren bunten Kleidern das Straßenbild beleben. Sie verkaufen den Touristen auf den Straßen und in den Restaurants selbstgefertigte Halsketten, Armbänder, verzierte Bogen und Pfeile, Blasrohre, deren Länge den Koffern der Reisenden bestens angepaßt sind. Die Indios leben in ihren Dörfern in der näheren und weiteren Umgebung Pucallpas und betreiben vorwiegend Landwirtschaft. Sie haben traditionell auch keine städtische, sondern eine ländliche Kultur.

Wo und wie wohnen die Kreolen und Mestizen in Pucallpa? Nun, ihrem Einkommen und ihrem sozialen Status entsprechend: Die Kreolen in Villen mit Vorgärten in der Stadt oder am Stadtrand, in Stadthäusern oder - eher selten - in Hotels (viele südamerikanische Großgrundbesitzer haben nämlich die $\mathrm{Ge}$ pflogenheit, während des (Süd-)Winters in den Städten zu leben). Die Mestizen leben ihrem niedrigeren Einkommen entsprechend in einfacheren Unterkünften. Vielfach sind es Bretterbuden mit Wellblechdächern, die in zwei oder mehrere Räume unterteilt sind und häufig weder über Trinkwasser noch Kanalisation verfügen.

Da die Bevölkerung Pucallpas sehr rasch wächst, ist die Stadt schon längst über die auf dem Stadtplan verzeichneten Grenzen hinausgewachsen. Deshalb gibt es rund um die Stadt herum eine wilde, spontane Besiedlung durch Mestizen und Indios. Solche Siedlungen ohne Trinkwasseranschluß, ohne Kanalisation und Elektrizität werden «pueblos jovenes» (Slums) genannt. Der Bevölkerungsdruck ist offenbar so groß, $\mathrm{da} ß$ es sogar im Hafenbecken von Pucallpa, das während der Trockenzeit fast ausgetrocknet ist, zur wilden, spontanen Besiedlung kommt.

\section{Pucallpa als zentraler Ort}

In Pucallpa wird eine fast unüberschaubare Vielzahl von Waren und Dienstleistungen angeboten. Es sind dabei alle Wirtschaftssektoren vertreten: Das Gewerbe 
bietet nicht nur Güter des täglichen Bedarfs an (Bäkkereien, Metzgereien, Supermarkt und offene, freie Märkte), sondern auch jene des mittleren und langfristigen Bedarfs (Kleider, Tuchhandlungen, Möbel, Haushaltapparate usw.). Im Handel sind zahlreiche Agenturen und Filialen von nationalen und internationalen Unternehmungen vertreten (Banken, Versicherungen, Transportunternehmungen usw.). Es gibt Industriebetriebe (Raffinerie, Brauerei, Sperrholzfabrik usw.), Erziehungs- und Gesundheitseinrichtungen, öffentliche und private Verwaltungen (Stadt-, Hafen-, Flughafen-, Postbehörden und von Busunternehmungen). Es gibt ein Gastgewerbe, eine Hotellerie und sogar zwei Kinos. Pucallpa ist Endpunkt einer der wichtigsten Strassen in Ostperu. Die Stadt verfügt über einen wichtigen Flußhafen und einen Flugplatz von nationaler Bedeutung.

Von dieser Vielzahl von Geschäften fallen vor allem die zahlreichen Eisenwarenhandlungen, Schlossereien, Schmieden, Autoreparaturwerkstätten, Spenglereien, Sanitärinstallations- und Elektrikergeschäfte, Schreinereien, Zimmereien usw. auf. Dieses nicht nur scheinbare, sondern offensichtliche Überangebot und breite Spektrum von Waren und Dienstleistungen dient nicht allein der Deckung des städtischen Bedarfs, sondern vielmehr der Versorgung der ganzen umliegenden Pionierzone. Pucallpa weist durch dieses reichhaltige Überangebot an Waren und Dienstleistungen in Ostperu einen hohen Zentralitätsgrad auf.

Der Ucayali ist während des ganzen Jahres von Schiffen mit einer Wasserverdrängung von etwa $3000 \mathrm{t}$ befahrbar, in der Trockenzeit für Schiffe mit über fünf Fuß Tiefgang. «In Pucallpa hat der Ucayali, selbst während der Trockenzeit neun Fuß Tiefe.»2) Pucallpa liegt etwa 1000 Flußkilometer von Iquitos und etwa 4500 Flußkilometer vom Atlantik entfernt. Schiffe bestimmter Unternehmungen fahren über diese Strecke sogar bis nach New York und Großbritannien. Die hauptsächlichsten auf den Schiffen transportierten Güter sind (nach maAs): Holz, Baumwolle, Reis, Bananen, Barbasco, Rohgummi und Rohöl. Das Rohöl kommt dabei vom Ölfeld von Ganso Azul (am Rio Pachitea) in die Raffinerien von Pucallpa und flußabwärts nach Iquitos (und früher auch nach Manaus). Die anderen Güter werden auf dem Wasserweg nach Pucallpa transportiert und dort entweder verarbeitet und vermarktet oder auf dem Landweg Richtung Lima weitertransportiert.

\section{Die wirtschaftliche Bedeutung der Stadt und deren historische Ursachen}

Pucallpa ist Zentrum einer Pionierzone. Die wirtschaftliche Bedeutung der Stadt ist mit der Geschichte der Kolonisationsbewegungen und dem Bau einer Straße, der «Carretera Central», verbunden.

Jede peruanische Regierung ist durch das starke Bevölkerungswachstum gezwungen (1970-1975 = 3,1\%), um die Selbstversorgung zu garantieren und neue Ar- beitsplätze zu schaffen, unter anderem die landwirtschaftliche Produktion zu steigern. Eine reale Steigerung der landwirtschaftlichen Produktion kann aber mit dem Bevölkerungswachstum nur Schritt halten, wenn die vorhandenen Reserven voll ausgeschöpft werden, wenn die Produktion gesteigert wird und wenn zusätzlich die Anbaufläche um ein Vielfaches erweitert wird. Eingehende Untersuchungen der Regierung hatten ergeben, daß die Erschließungskosten für den Anbau landwirtschaftlicher Produkte gegenüber den anderen Regionen des Landes (Costa und Sierra) im Oriente am geringsten sind. Deshalb entschlossen sich die verschiedenen peruanischen $\mathrm{Re}$ gierungen mit mehr oder weniger großem Erfolg, den noch relativ «unerschlossenen» Osten (oriente) zu kolonisieren, um dort erstens Neuland für die Landund Forstwirtschaft zu gewinnen, und zweitens um dort nach neuen Rohstoffquellen (Minerale, Erdöl, Erdgas usw.) zu suchen.

Trotz zahlreicher Vorteile sind mit der Kolonisierung des Oriente auch viele Nachteile verbunden, so etwa die fehlende oder nur mangelhaft ausgebaute Infrastruktur. Daran sind schon zahllose Kolonisierungsbemühungen gescheitert. Pucallpa besitzt zwar einen wichtigen Flußhafen, der auch mit größeren Schiffen erreichbar ist, jedoch entwässert der Ucayali über den Amazonas in den 4500 Flußkilometer entfernten Atlantik. Auf der viel kürzeren Strecke, etwa zwischen Pucallpa und Lima (Luftdistanz ca. $875 \mathrm{~km}$ ) gibt es keine natürliche Verbindung, denn die Anden liegen dazwischen. Für die wirtschaftliche Erschließung des Oriente und dessen politische Integration ist es deshalb unerläßlich, die Infrastruktur auszubauen.

Daher kamen bereits 1851 im allgemeinen Eisenbahnfieber Pläne auf, eine Bahnlinie zu bauen, die bei Tambo-del-Sol (in den Anden: km 312 [von Lima]; Höhe $4112 \mathrm{~m}$ ) von der Cerro-de-Pasco-Bahn in östlicher Richtung über Oxapampa und Pozuzo hätte geführt werden und nach etwa $580 \mathrm{~km}$ Pucallpa erreichen, und damit eine Eisenbahnverbindung zwischen Lima (oder besser deren Hafenstadt Callao) und Pucallpa hätte herstellen sollen. Von diesem Plan wurden im Hochland aber lediglich $78 \mathrm{~km}$ realisiert: Die Bahnlinie endet etwa $16 \mathrm{~km}$ hinter Huachon, wobei das Trasse aber noch ungefähr $40 \mathrm{~km}$ weiterführt. Von Pucallpa aus wurden zwischen 1921 und 1929 etwa $11 \mathrm{~km}$ Schienen verlegt, berichtet MAAS weiter. Mit der aufkommenden Motorisierung wurden aber die Pläne zum Bau der Eisenbahnlinie zwischen Tambodel-Sol und Pucallpa Ende der zwanziger Jahre wieder aufgegeben und durch einen neuen Plan zum Bau einer Autostraße ersetzt. Die in den zwanziger Jahren realisierten Streckenabschnitte der Eisenbahnlinie sind heute außer Betrieb oder, wie im Falle von Pucallpa, wurde jene Teilstrecke von $11 \mathrm{~km}$ wieder demontiert und das Material für den Straßenbau (Brücken usw.) verwendet.

Eine Straßenverbindung zwischen Lima und den Anden bestand bereits, aber keine Fortsetzung in den 


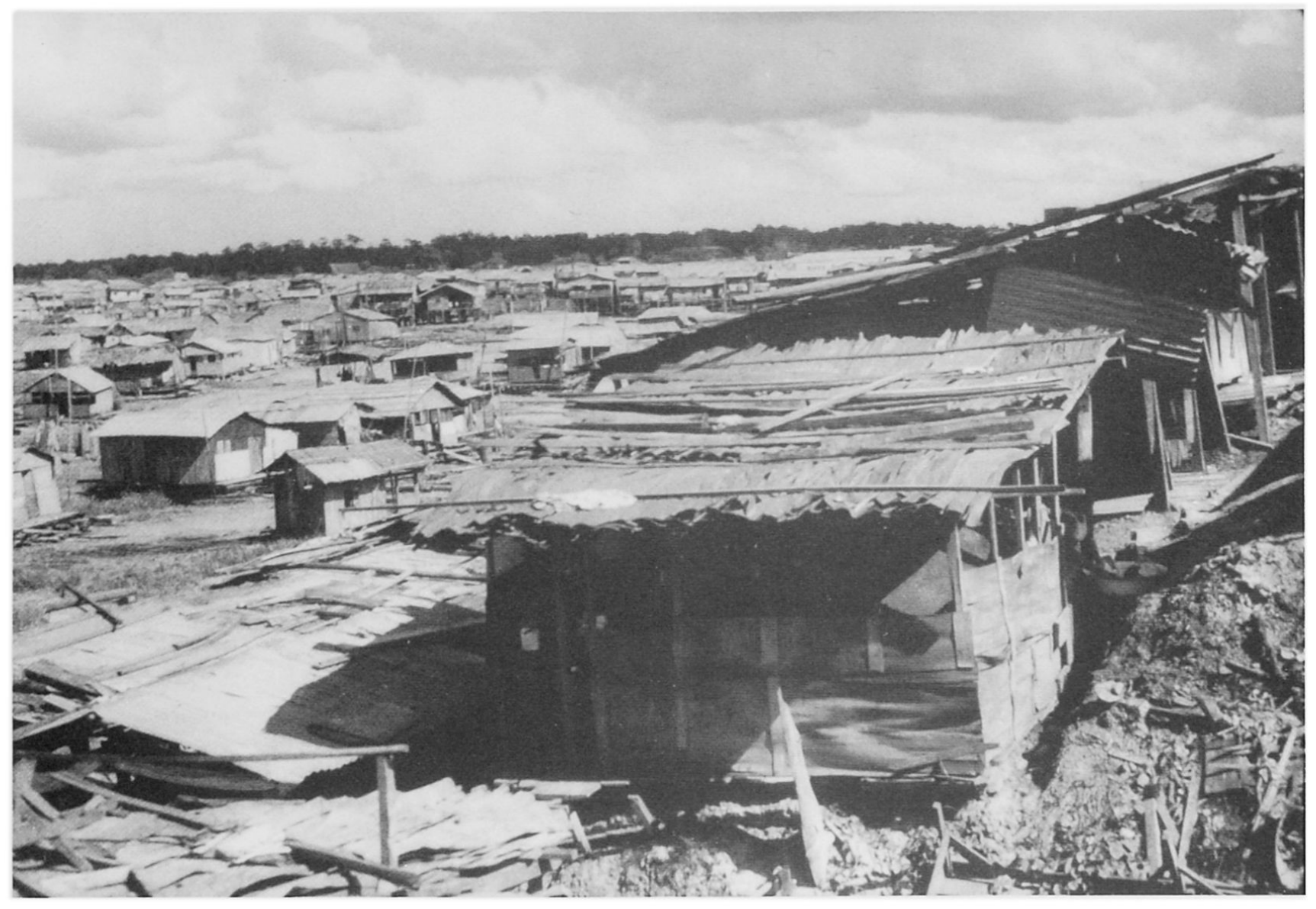

Abb. 4: «Da die Bevölkerung Pucallpas sehr rasch wächst, ist die Stadt schon längst über die auf dem Stadtplan verzeichneten Grenzen hinausgewachsen. Deshalb gibt es rund um die Stadt herum eine wilde, spontane Besiedlung durch Mestizen und Indios. Solche Siedlungen ohne Trinkwasseranschluß, ohne Kanalisation und Elektrizität werden "pueblos jovenes" (Slums) genannt. Der Bevölkerungsdruck ist offenbar so groß, daß es sogar im Hafenbecken von Pucallpa, das während der Trockenzeit fast ausgetrocknet ist, zur wilden, spontanen Besiedlung kommt.”

Oriente. Und so ging es also darum, die «Carretera Central», wie diese Pionierstraße genannt wird, um etwa $300 \mathrm{~km}$ bis nach Pucallpa zu verlängern. Mit dem Bau der Straße wurde 1933 begonnen. Sie erreichte 1936 Tingo Maria (ebenfalls ein wichtiges Pionierzentrum) und, nach Überwindung verschiedener Schwierigkeiten, 1942 Pucallpa am Ucayali. Offiziell wurde die Straße am 8. September 1943 dem Verkehr übergeben. Pucallpa war damit über eine Strecke von $843 \mathrm{~km}$ mit Lima an der Pazifikküste verbunden.

Diese Straße ist in ihrer Art für Peru einzigartig, und zwar deshalb, weil mit ihr zum ersten Mal in der Geschichte dieses Landes eine direkte Verbindung zwischen der Pazifikküste und einem auch mit größeren Schiffen erreichbaren Flußhafen im Amazonasflußsystem hergestellt wurde. Weitere Straßen sind in verschiedenen Pioniergebieten zwar geplant, unter anderem auch die "Carretera Marginal de la Selva", die verschiedene Anrainerstaaten am Andenostfuß miteinander verbinden soll. Jedoch ist die «Carretera Central» die einzige bisher fertiggebaute und seit etwa 1969 auch asphaltierte Straße. Und als einzige ist sie deshalb von ungeheurer wirtschaftlicher Bedeutung. Ein großer Teil der Versorgung hängt von der Existenz dieser Straße ab. Auch nur kurze Unterbrechungen bringen schon massive wirtschaftliche Einbußen im gesamten Güteraustausch.

Von ihrer wirtschaftlichen Bedeutung her müßte man eigentlich annehmen, daß die Straße entsprechend wetterfest gebaut worden ist. Jedoch ist man erstaunt, wenn man sieht, in welch schlechtem Zustand sie ist. Beim Bau wurde offenbar bloß ein Asphaltbelag über den unstabilen, sandigen Untergrund gelegt. Schon bei kurzen, aber sehr starken tropischen Regengüssen wird die Straße unterspült, wobei dann natürlich der Asphaltbelag zusammenfällt. Auch wenn die Straße «normal» befahrbar ist, kann man auf gewissen Strecken nicht schneller als $5 \mathrm{~km} / \mathrm{h}$ fahren. Aus Geldmangel wurde die Straße offenbar nie fachmännisch geplant und ausgeführt. Auch die Reparaturen bleiben aus Geldmangel in oberflächlicher Kosmetik stecken. Solche Infrastrukturprobleme zeigen augenscheinlich die ökologischen Grenzen einer ungehemmten, im Stil der Kolonialzeit verlaufenden Wirtschaftsentwicklung auf.

Die große Bedeutung der Straße für Pucallpa und deren umliegende Pionierzone zeigt sich sehr deutlich in deren Bevölkerungsentwicklung. 


\section{Bevölkerungsentwicklung}

Pucallpa hat heute nach inoffiziellen Schätzungen etwa 70000 Einwohner und ist - auch in bezug auf seine zentralörtliche Stellung - nach Iquitos der zweitgrößte Ort des ostperuanischen Departementes Loreto. Das war nicht immer so. Wie MAAS berichtet, fand die offizielle Stadtgründung im Jahre 1896 «im Stil der Kolonialzeit» statt. Pucallpa bestand damals lediglich aus «ein paar armseligen Strohhütten, die auseinandergezogen auf dem hohen Westufer des Ucayali lagens. Pucallpa war also auch durch den offiziellen Akt der Stadtgründung noch ein unbekannter und bedeutungsloser Flecken ohne Straßenverbindung ins Hinterland, ein Ort also wie es ihn zu Hunderten im Amazonastiefland gibt.

1912 hatte Pucallpa 50 Einwohner. Viele von ihnen hatten sich dort niedergelassen, weil sie hofften, daß eine geplante Eisenbahnlinie gebaut würde. «1920 kamen 150 Arbeiter hinzu, die mit der Vorbereitung der Eisenbahntrasse und dem Verlegen der Schienen beschäftigt wurden. Außerdem lebten zu jenem Zeitpunkt in der Umgebung schätzungsweise 3000 Einzelsiedler von Subsistenzanbau, Kautschukzapfen, Jagd und Fischfang. Obwohl der Eisenbahnbau wieder aufgegeben wurde, wohnten 1940 bereits 2368 Menschen in Pucallpa selbst, dagegen nur noch 2552 in der Umgebung.» Dieses Anwachsen der Bevölkerung Pucallpas und die gleichzeitige Abnahme jener in der Umgebung erklärt sich MAAS daraus, daß eine starke Konzentration von Einzelsiedlern in der Stadt stattgefunden hatte. $\mathrm{Zu}$ jenem Zeitpunkt ließen sich viele in der Hoffnung auf den bevorstehenden Bau der «Carretera Central» in Pucallpa nieder, um dadurch Arbeit und Land zu erhalten.

Verfolgt man die Bevölkerungsentwicklung in den darauffolgenden Jahren weiter, so sieht man, daß der entscheidende Wendepunkt in der Entwicklung beim Ende des Baues (1942) und der offiziellen Eröffnung der «Carretera Central» im Jahre 1943 liegt. Die Bevölkerung der Stadt nahm zwar weiterhin zu, jedoch bedeutete die Existenz der Straße das Einleiten einer noch heute andauernden Kolonisierungsbewegung in die Pionierzone um Pucallpa herum, insbesondere entlang der Pionierstraße. 1955, also 13 Jahre nach der Eröffnung der Straße, zählte Pucallpa 15000 Einwohner, dagegen lebten in der umliegenden Pionierzone bereits 31000 Menschen. Das heißt, die Bevölkerung in der Stadt wuchs seit 1940 um etwa 500\%, jene in der Umgebung jedoch um $1100 \%$.

«Die Volkszählung von 1961 ergab für das Siedlungsgebiet Pucallpa und das Gebiet an den stromaufwärts gelegenen Zuflüssen (Provinz Coronel Portillo) eine Bevölkerungsziffer von 64161 und die Fortschreibung zum 30.6.1966 ca. 88000 Einwohner. Im Dezember 1966 hatte die Stadt Pucallpa nach amtlicher Fortschreibung 25000 Einwohner, doch wurde inoffiziell eine Bevölkerung von ca. 40000 geschätzt.» (A. MAAS). Man kann annehmen, daß diese Entwicklung bis heute anhält, allerdings nicht mehr in so rasantem Tempo wie man das vielleicht noch vor zehn Jahren angenommen hatte, und zwar deshalb, weil sich bei vielen Kolonisationsprojekten die hochgesteckten Erwartungen nicht erfüllten.

Am erstaunlichsten ist diese Bevölkerungsentwicklung, wenn man bedenkt, daß es in Pucallpa bis 1967 weder Trinkwasser noch Kanalisation gab. Die Straßen in der Stadt sind bis heute noch nicht asphaltiert und die «Carretera Central» zwischen Pucallpa und Tingo Maria erst seit 1969. Einer Radiomeldung vom 20. Oktober 1978 zufolge riefen die Gewerkschaften Pucallpas während eines Generalstreikes die Zentralbehörden in Lima unter anderem auf, die Straßen - oder zumindest die Hauptstraßen - nun endlich zu asphaltieren.

\section{Der Pioniergeist: Das Denken im Präsens}

Das Erscheinungsbild Pucallpas hinterläßt insgesamt den Eindruck, als hätten die "colonos» kein großes Vertrauen in die Zukunft, sondern daß sie für den Augenblick lebten. Oder noch extremer: daß sie jetzt überleben wollen, denn wer weiß, was morgen sein wird. Sie sind deshalb krampfhaft und mit allen legalen und illegalen Mitteln um die Gegenwart bemüht, um soviel als möglich vom «Kuchen» abzubekommen, denn so viele «Kuchen» gibt es in einem Entwicklungsland, wie Peru eines ist, gar nicht. Dieses ausschließlich auf die Gegenwart bezogene Denken kommt allerdings nicht von ungefähr, denn wie oft schon in der Kolonisationsgeschichte des Amazonastieflandes, etwa zur Zeit des Kautschukbooms oder des Rosenöls, mußten die Leute erleben, wie schnell der Traum vom großen Geld ausgeträumt war. Letzter sichtbarer Zeuge jener "großen Zeit» ist Manaus am Amazonas: Die vom einstigen Ruhm und Glanz zeugende Oper vergammelt unter der tropischen Sonne. Das Leben ist nur allzu kurz und verlangt darum einen hohen Einsatz. Nur wer bis zum Äußersten kämpft, überlebt, die anderen, die Schwachen gehen zugrunde - auch ökonomisch. Der Pioniergeist, der Pucallpa beherrscht, ist jener «nackte» Kapitalismus, der den Beginn der Industrialisierung in Europa kennzeichnete: Es herrscht der reinste Sozialdarwinismus, wie ihn schon HERBERT SPENCER treffend charakterisierte: «This survival of the fittest».

\section{Anmerkungen:}

1) WILHELMY H.: Südamerika im Spiegel seiner Städte, Hamburg 1952.

2) MAAS A.: Entwicklung und Perspektiven der wirtschaftlichen Erschließung des tropischen Waldlandes von Peru, unter besonderer Berücksichtigung der verkehrsgeographischen Problematik, Tübinger Geogr. Studien, 1969. 